\title{
Stellar ages, masses, and radii from asteroseismic modeling are robust to systematic errors in spectroscopy (Corrigendum)
}

\author{
E. P. Bellinger ${ }^{1}$, S. Hekker ${ }^{2,1}$, G. C. Angelou ${ }^{3}$, A. Stokholm ${ }^{1}$, and S. Basu ${ }^{4}$ \\ ${ }^{1}$ Stellar Astrophysics Centre, Department of Physics and Astronomy, Aarhus University, Aarhus, Denmark \\ e-mail: bellinger@phys.au.dk \\ 2 Stellar Ages \& Galactic Evolution (SAGE) Group, Max Planck Institute for Solar System Research, Göttingen, Germany \\ 3 Max Planck Institute for Astrophysics, Garching, Germany \\ 4 Department of Astronomy, Yale University, New Haven, CT, USA
}

A\&A, 622, A130 (2019), https://doi .org/10 . 1051/0004-6361/201834461

Key words. asteroseismology - stars: abundances - stars: low-mass - stars: evolution - stars: oscillations - errata, addenda

The original article presented the density scaling relation (Eq. (2)) incorrectly. The correct relation is:

$\frac{\rho}{\rho_{\odot}} \simeq\left(\frac{\Delta v}{\Delta v_{\odot}}\right)^{2}$

This error affects the rightmost panel of Fig. 10, the bottom left panel of Fig. 13, and the bottom left panel of Fig. 14. The corrected panels are presented in Figs. 1 and 2.

The sentence "We note that the large number of outliers in mean density estimates in Fig. 10 arise mainly from their very small uncertainties". is no longer relevant, as the majority of these are no longer outliers with the correct relation.

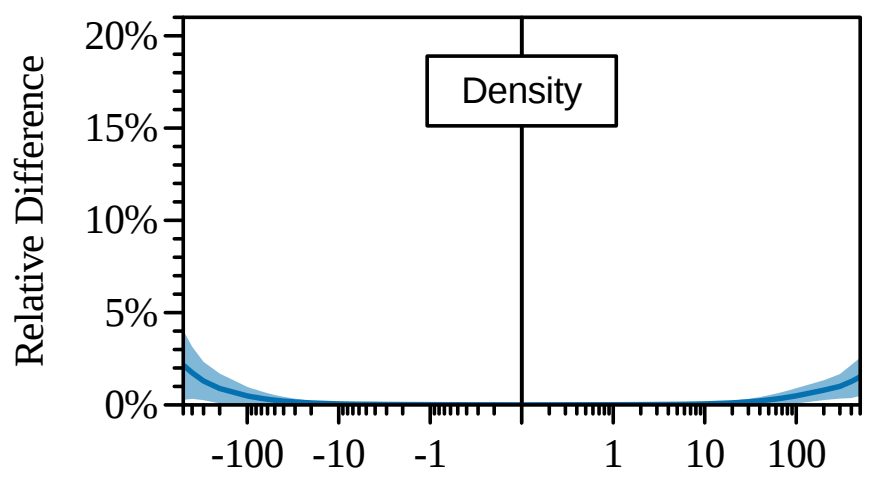

Systematic Error [K]

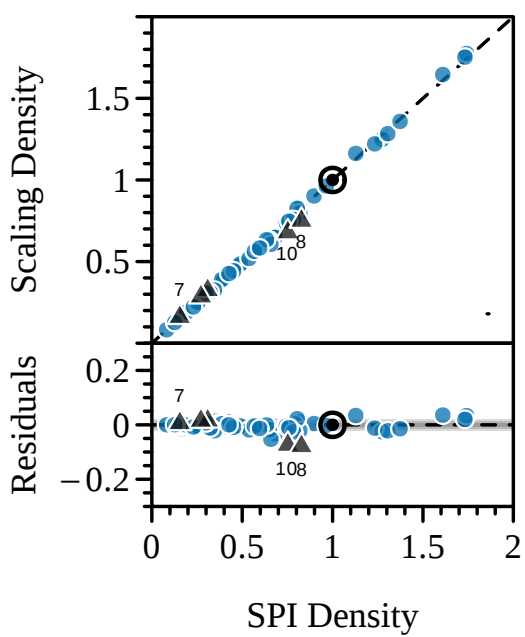

Fig. 1. Corrected rightmost panel of Fig. 10. The biases in the residuals are consistent with zero $\left(-0.004 \pm 0.017 \rho_{\odot}\right)$.

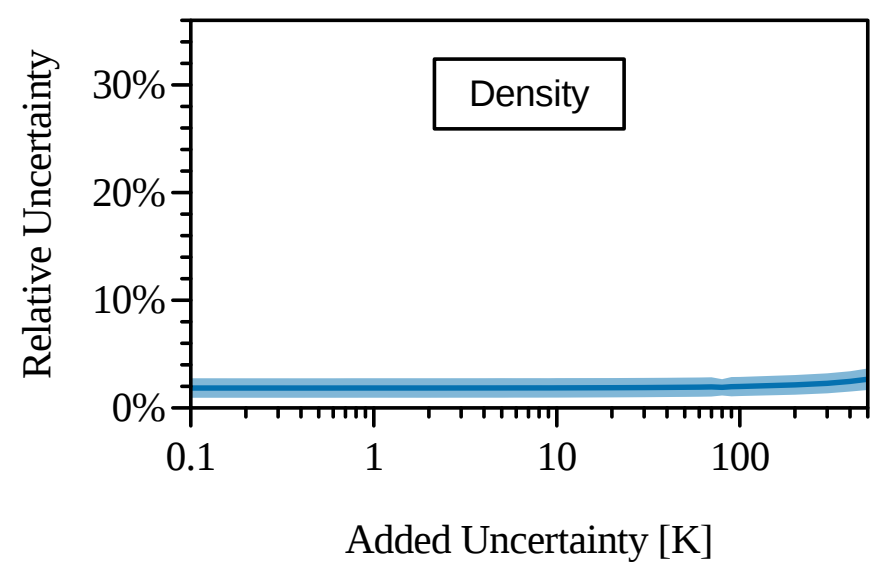

Fig. 2. Corrected bottom left panels of Figs. 13 and 14. The dashed lines corresponding to the scaling relations have been removed. 